\title{
Typology of eaters based on conventional and organic food consumption: results from the NutriNet-Santé cohort study
}

\author{
Julia Baudry $^{1 *}$, Mathilde Touvier ${ }^{1}$, Benjamin Allès ${ }^{1}$, Sandrine Péneau ${ }^{1}$, Caroline Méjean ${ }^{1}$, Pilar Galan ${ }^{1}$, \\ Serge Hercberg ${ }^{1,2}$, Denis Lairon ${ }^{3}$ and Emmanuelle Kesse-Guyot ${ }^{1}$ \\ ${ }^{1}$ COMUE Sorbonne-Paris-Cité, Equipe de Recherche en Epidémiologie Nutritionnelle (EREN), Centre d'Epidémiologie et \\ Statistiques Paris Cité, Institut National de la Santé et de la Recherche Médicale (INSERM, U1153), Institut National de la \\ Recherche Agronomique (INRA, U1125), Conservatoire National des Arts et Métiers (CNAM), Université Paris 13, F-93017 \\ Bobigny, France \\ ${ }^{2}$ Département de Santé Publique, Hôpital Avicenne, F-93017 Bobigny, France \\ ${ }^{3}$ Nutrition, Obésité et Risque Thrombotique (NORT), Institut National de la Santé et de la Recherche Médicale (INSERM), UMR S \\ 1062, Institut National de la Recherche Agronomique (INRA) 1260, Aix Marseille Université, 13005 Marseille, France
}

(Submitted 11 February 2016 - Final revision received 13 May 2016 - Accepted 20 May 2016 - First published online 17 June 2016)

\begin{abstract}
Limited information is available on large-scale populations regarding the socio-demographic and nutrient profiles and eating behaviour of consumers, taking into account both organic and conventional foods. The aims of this study were to draw up a typology of consumers according to their eating habits, based both on their dietary patterns and the mode of food production, and to outline their socio-demographic, behavioural and nutritional characteristics. Data were collected from 28245 participants of the NutriNet-Santé study. Dietary information was obtained using a 264-item, semi-quantitative, organic FFQ. To identify clusters of consumers, principal component analysis was applied on sixteen conventional and sixteen organic food groups followed by a clustering procedure. The following five clusters of consumers were identified: (1) a cluster characterised by low energy intake, low consumption of organic food and high prevalence of inadequate nutrient intakes; (2) a cluster of big eaters of conventional foods with high intakes of SFA and cholesterol; (3) a cluster with high consumption of organic food and relatively adequate nutritional diet quality; (4) a group with a high percentage of organic food consumers, $14 \%$ of which were either vegetarians or vegans, who exhibited a high nutritional diet quality and a low prevalence of inadequate intakes of most vitamins except $\mathrm{B}_{12}$; and (5) a group of moderate organic food consumers with a particularly high intake of proteins and alcohol and a poor nutritional diet quality. These findings may have implications for future aetiological studies investigating the potential impact of organic food consumption
\end{abstract}

Key words: Nutrient intakes: Profiles of consumers: Clusters: Organic foods: Dietary patterns

Concurrently with the increase in demand for organic food worldwide $^{(1)}$, the last two decades have seen a growing number of research studies investigating the characteristics of organic food consumers ${ }^{(2-15)}$. However, research on organic food consumption to date has largely focused on differences between frequent users and non-users. Numerous studies have thus investigated the relationships between frequency of organic food consumption/purchase and socio-demographic and lifestyle factors as well as their associated food patterns ${ }^{(3,6,9,12,15)}$. These studies have suggested that frequent organic food consumption is associated with a higher level of education, vegetarianism or healthier lifestyle and dietary patterns. In these studies, regular or non-organic food users are often considered in single broad categories reflecting the overall frequency of purchase only. These approaches do not take into account the variety of nutritional behaviours and the relative share of organic food intake in the diet. It is, however, likely that the potential effects of an organic diet are associated with the type (i.e. food groups) and the quantity of organic food consumed.

To our knowledge, only one study conducted on males in Germany has attempted to establish a typology of organic and non-organic food purchasers based on their actual consumption of meat, fruits and vegetables ${ }^{(14)}$. However, in this analysis, men were identified as either organic food purchasers or non-organic food purchasers on the basis of their own statement regarding their purchase of organic food. For future aetiological studies on organic food consumption, a precise understanding of the diversity of profiles taking into account both dimensions (i.e. type and mode of production of the food consumed) is necessary. Indeed, assuming that conventional

Abbreviations: mPNNS-GS, modified Programme National Nutrition Santé Guidelines Score; Org-FFQ, organic FFQ.

* Corresponding author: J. Baudry, email j.baudry@eren.smbh.univ-paris13.fr 
(i.e. non-organic) food consumption is systematically associated with a poor nutritional quality of diet and conversely for an organic-based diet would probably be a simplistic approach in health studies.

Therefore, the aims of the present study were to identify a typology of consumers on the basis of a detailed estimation of the consumption of organic and conventional foods in a large sample of French adults from the NutriNet-Santé cohort study and to describe these consumers' groups in terms of sociodemographic, lifestyle, behavioural and nutritional profiles.

\section{Methods \\ Study population}

The NutriNet-Santé study is an ongoing, observational, prospective, open-cohort study, which was launched in 2009 in France $^{(16)}$. The study aims to investigate the relationships between nutrition and health as well as the determinants of dietary patterns and nutritional status. To be included in the cohort, participants must complete a baseline set of selfadministered, web-based questionnaires on dietary intake, as well as health and anthropometric, socio-demographic and lifestyle characteristics. During follow-up, participants were also invited to complete extensive questionnaires related to determinants of dietary behaviour, as well as nutritional and health-related characteristics.

\section{Ethics}

This study was conducted in accordance with the Declaration of Helsinki, and all procedures were approved by the Institutional Review Board of the French Institute for Health and Medical Research (IRB INSERM no. 0000388FWA00005831) and the Commission Nationale de l'Informatique et des Libertés (CNIL no. 908450 and no. 909216). All participants provided their informed consent with an electronic signature, and this study is registered in EudraCT (European Union Drug Regulating Authorities Clinical Trials; no. 2013-000929-31).

\section{Data collection and treatment}

Dietary intake. To assess usual dietary consumption, participants reported the frequency and portion sizes of consumed foods and beverages by filling out a dedicated 264-item, semiquantitative, organic FFQ (Org-FFQ). The Org-FFQ was based on a validated FFQ supplemented by a section pertaining to the frequency of organic food consumption (it should be noted that this latter part was not validated) ${ }^{(17)}$. The Org-FFQ has been described elsewhere ${ }^{(18)}$. In brief, subjects were asked to report their frequency of consumption and the quantity consumed over the past year for each of the 264 items. In addition, a five-point likert-type scale ranging from never to always was used to estimate the frequency of organic food consumption of each food item. For each food item, the question was as follows: How often was the product of organic origin? The term 'organic' referred to European Union-certified organic products. Organic food intake was obtained for each item by applying a weight of $0,0.25,0.5,0.75$ and 1 to the five respective categories of frequency (never, rarely, half the time, often and always). Beverage and food items were aggregated into sixteen food groups. The average total daily intake in grams per day of the sixteen food categories was assessed. We separated conventional from organic intake for each food group on the basis of the relative share reported. In other words, for each food group, we calculated organic food intake on the basis of the relative share reported, and conventional food intake was obtained by subtracting the total food intake from the organic food intake.

The proportion of organic food in the whole diet was computed by averaging the total organic food intake $(\mathrm{g} / \mathrm{d})$ out of the total intake excluding water $(\mathrm{g} / \mathrm{d})$ multiplied by 100 .

To estimate nutrient intakes, an ad hoc food composition database (regardless of the origin of the products) was developed for the Org-FFQ using the original NutriNet-Santé composition table ${ }^{(19)}$. As the origin (organic or conventional) of foods was not taken into account, some potential differences in contents reported in the literature resulting from the mode of production were not considered ${ }^{(20-22)}$.

We also computed two a priori nutritional diet quality scores: the modified Programme National Nutrition Santé Guidelines Score (mPNNS-GS), which is a thirteen-five-point score that reflects adherence to the French nutritional recommendations ${ }^{(23)}$, and the PANDiet, a 100-point score that reflects the probability of adequate nutrient intake ${ }^{(24)}$. The prevalence of inadequate intakes of selected nutrients was estimated by the proportion of subjects with intake below the estimated average requirement for the French population ${ }^{(25,26)}$.

Socio-demographic, lifestyle and behavioural characteristics. Upon enrolment and each year thereafter, participants provided information on socio-demographic and lifestyle characteristics including sex, age, education, income, number of children, marital status, place of residence, smoking status and practice of vegetarian or vegan diets. A vegetarian diet was defined as a diet that did not include any meat (the term 'meat' included red meat, processed meat and poultry), whereas a vegan diet was defined as a diet that excluded all foods of animal origin (exclusion of meat, seafood, eggs or dairy products). The question was as follows: Are you currently following a particular diet? Yes, I am a vegetarian (I do not eat any meat but I can eat products of animal origin). Yes, I am a vegan (I do not eat any meat, seafood, eggs or dairy products).

The closest available data to the Org-FFQ questionnaire completion date were used. Monthly household income was calculated by household units (HU): $1 \mathrm{HU}$ was attributed for the first adult in the household, 0.5 for other persons aged 14 years or older and 0.3 for children under 14 years ${ }^{(27)}$.

In July 2014, participants were also invited to fill out a questionnaire pertaining to attitudes, perceptions and motivations towards organic food. In particular, subjects were asked to self-report their frequency of organic food consumption (regular/occasional/non-consumption) and to identify the official organic labels among twelve different logos. For a list of conventional and organic food categories, subjects were also asked to report their main place of purchase from among the different alternatives provided. They could also declare that they did not consume the product. For each place of purchase, 
a score (in \%) was calculated by adding up the number of times this source of supply was selected as a main place of purchase for the different foods and dividing by the number of foods purchased by the participant. Subjects were also asked to provide information concerning whether they engaged (or not) in selected environmentally friendly practices (i.e. practices aimed at reducing consumption of energy and production of waste). The questions were as follows: (1) Did you consider the energy performance label when purchasing your refrigerator? (2) Do you regularly defrost your refrigerator? (3) Do you use a lid when heating up water (4) Do you recycle organic waste?

\section{Statistical analyses}

The Org-FFQ, an optional questionnaire, was administered over a 5-month period from June to October 2014 to the subjects included in the cohort at that time - that is, 104080 participants. Of the subjects who completed the Org-FFQ ( $n$ 33384), a total of 28245 were selected (20980 women and 7265 men). We excluded under-reporting or over-reporting participants with implausible energy intakes ( $n$ 2097), participants with missing covariates ( $n$ 2320) or those living overseas $(n$ 722). To better characterise the selected sample, we compared participants who completed the Org-FFQ with excluded NutriNet-Santé participants using $\chi^{2}$ tests and Student $t$ tests, as appropriate.

Concerning questions related to recognition of labels, selfreported organic food consumption and environment-friendly practices, a sub-sample was considered with no missing information in terms of these variables. The sample size was thus 23010. Likewise, for the distribution of places of purchase, the sample size was 22985 .

Under- and over-reporting participants were identified on the basis of their 'energy intake:energy requirement' ratio. Individuals with ratios below or above specific cut-off values previously identified in the FFQ were excluded ${ }^{(17)}$. BMR was estimated using Schofield equations ${ }^{(28)}$.

To identify different clusters of consumers of conventional and organic products, principal component analysis (PCA) was applied on the sixteen conventional and sixteen organic food group intakes (in $\mathrm{g} / \mathrm{d}$, thirty-two variables), thereby taking into account the type of food and the mode of food production (organic or conventional). This method allows the reduction of the initial range of information by maximising variance. In total, six dimensions were retained according to eigenvalues $>1$, scree test and interpretability of factors ${ }^{(29)}$. We then performed a two-step clustering procedure by applying Ward's hierarchical clustering using the first six dimensions retained in the PCA, followed by a non-hierarchical, K-means clustering procedure based on the earlier hierarchical clustering. PCA was performed using SAS ${ }^{\circledR}$ PROC PRINCOMP, and the clustering procedure was performed using SAS $^{\circledR}$ PROC CLUSTER and PROC FASTCLUS (version 9.4; SAS Institute Inc.) ${ }^{(29)}$.

We described the clusters in terms of socio-demographic and lifestyle characteristics, dietary profiles and prevalence of inadequate intakes, purchasing behaviours and environmentally friendly practices.

Associations of clusters with the above-listed characteristics were assessed by ANCOVA. We provide mean values and
95\% CI. Dietary characteristics were adjusted for sex, age and total energy intake, and average percentages of place of purchase were adjusted for sex and age. For categorical variables, the $P$ value was based on $\chi^{2}$ test.

Tests of statistical significance were two-sided, and type I error was set at $5 \%$. Statistical analyses were performed using SAS software (version 9.4; SAS Institute Inc.).

\section{Results}

The proportion of men was significantly higher among respondents to the Org-FFQ than among participants excluded from the analysis ( $26 v .21 \%$ ). The respondents were also older than the excluded subjects (53.20 (SD 14.07) v. 44.62 (SD 14.20) years old) (data not tabulated).

\section{Cluster description}

On the basis of the two-step clustering procedure described above, five clusters were identified, representing, respectively, $39.0 \%$ (cluster 1), 20.6\% (cluster 2), 24.8\% (cluster 3), 10.8\% (cluster 4 ) and $4.8 \%$ (cluster 5 ) of the study population. For all variables, $P$ values were $<0 \cdot 0001$.

According to their main dietary traits, the different clusters are labelled as follows.

Cluster 1: standard conventional food small eaters. Subjects in this cluster were younger compared with other clusters (Table 1). The highest proportion of urban population was found in this group. They had low energy intake, low proportion of organic food in their diet and exhibited intermediate nutritional diet quality (intermediate values of PANDiet and mPNNS-GS scores) (Table 2). The subjects were, for the most part, those with the highest prevalence of inadequate intakes except for vitamin $\mathrm{B}_{12}$ and $\mathrm{Ca}$. Their low prevalence of inadequate intakes of $\mathrm{Ca}$ is noteworthy (Table 3). The most frequently cited main place of purchase by the subjects was supermarkets followed by retail stores and markets (Table 4).

Cluster 2: unhealthy conventional food big eaters. This group was composed of a third of men and characterised by the highest percentages of individuals with low income and educational levels (Table 1). They had the lowest organic food consumption with high energy intake and an overall poor nutritional diet quality (low PANDiet and mPNNS-GS) with high intakes of cholesterol and SFA (Table 2). For most nutrients, subjects had the lowest prevalence of inadequate intakes except for folate, vitamins $\mathrm{C}$ and $\mathrm{E}, \mathrm{Mg}, \mathrm{Fe}$ and $\mathrm{Ca}$ (Table 3). The most frequently reported main place of food purchase was the supermarket followed by retail stores and discount stores (Table 4).

Cluster 3: standard organic food small eaters. The lowest proportion of men was found in this cluster (Table 1). Subjects had the second highest proportion of organic food in their diet (Table 2). They had a relatively low energy intake and the second highest intake of fibre and PUFA (Table 2). They exhibited a relatively adequate nutritional diet quality 
Table 1. Characteristics of the participants, NutriNet-Santé study, 2014

(Numbers and percentages; mean values and standard deviations; $n$ 28245)

\begin{tabular}{|c|c|c|c|c|c|c|}
\hline & Cluster 1 & Cluster 2 & Cluster 3 & Cluster 4 & Cluster 5 & $P^{*}$ \\
\hline$n$ & 11.012 & $5 \cdot 811$ & 7.007 & 3.048 & $1 \cdot 367$ & \\
\hline$\%$ & $39 \cdot 0$ & $20 \cdot 6$ & $24 \cdot 8$ & $10 \cdot 8$ & $4 \cdot 8$ & \\
\hline $\operatorname{Sex}(\%)$ & & & & & & $<0.0001$ \\
\hline Female & 78.67 & $67 \cdot 70$ & $80 \cdot 22$ & $76 \cdot 28$ & 31.97 & \\
\hline Male & 21.33 & $32 \cdot 30$ & $19 \cdot 78$ & 23.72 & 68.03 & \\
\hline Age (years) & & & & & & $<0.0001$ \\
\hline Mean & $51 \cdot 72$ & 53.91 & $54 \cdot 48$ & 53.42 & $61 \cdot 18$ & \\
\hline SD & $14 \cdot 71$ & 14.05 & $13 \cdot 13$ & 13.53 & $11 \cdot 19$ & \\
\hline Educational level (\%) & & & & & & $<0.0001$ \\
\hline$<$ High school diploma & 21.47 & 26.07 & $20 \cdot 07$ & $17 \cdot 62$ & $25 \cdot 68$ & \\
\hline High school diploma & $15 \cdot 01$ & $16 \cdot 74$ & $15 \cdot 13$ & $13 \cdot 78$ & 13.97 & \\
\hline Post-secondary graduate & 63.52 & $57 \cdot 18$ & 64.81 & 68.60 & $60 \cdot 35$ & \\
\hline Monthly income per household unit (\%) & & & & & & $<0.0001$ \\
\hline Refuse to declare & $12 \cdot 76$ & $12 \cdot 30$ & 13.02 & $11 \cdot 38$ & $7 \cdot 17$ & \\
\hline $900-1200$ euros & 11.47 & $13 \cdot 23$ & $9 \cdot 01$ & $13 \cdot 19$ & $6 \cdot 51$ & \\
\hline $1200-1800$ euros & $21 \cdot 77$ & 23.83 & $20 \cdot 38$ & 21.95 & $17 \cdot 70$ & \\
\hline $1800-2700$ euros & $25 \cdot 19$ & $24 \cdot 68$ & $25 \cdot 77$ & $25 \cdot 59$ & $27 \cdot 87$ & \\
\hline$>2700$ euros & $28 \cdot 81$ & $25 \cdot 95$ & 31.83 & $27 \cdot 89$ & $40 \cdot 75$ & \\
\hline Location (\%) & & & & & & $<0.0001$ \\
\hline Rural community & $19 \cdot 81$ & $22 \cdot 44$ & 23.65 & 24.87 & 24.43 & \\
\hline Urban unit with a population $<20000$ inhabitants & $14 \cdot 79$ & $15 \cdot 49$ & $15 \cdot 83$ & $16 \cdot 50$ & $16 \cdot 75$ & \\
\hline Urban unit with a population between 20000 and 200000 inhabitants & $17 \cdot 79$ & $18 \cdot 38$ & 17.95 & $17 \cdot 88$ & $18 \cdot 07$ & \\
\hline Urban unit with a population $>200000$ inhabitants & $47 \cdot 60$ & 43.69 & 42.57 & $40 \cdot 75$ & $40 \cdot 75$ & \\
\hline Smoking status (\%) & & & & & & $<0.0001$ \\
\hline Never smokers & 52.03 & $47 \cdot 39$ & 48.89 & 49.93 & 23.77 & \\
\hline Former smokers & $36 \cdot 58$ & $41 \cdot 21$ & 41.46 & $40 \cdot 52$ & $56 \cdot 84$ & \\
\hline Current smokers & 11.39 & 11.39 & $9 \cdot 65$ & 9.55 & $19 \cdot 39$ & \\
\hline Vegan or vegetarian diet (yes) (\%) & 1.56 & 0.67 & 3.08 & 14.40 & 0.73 & $<0.0001$ \\
\hline Recognition of the French label (yes) (\%)† & 92.58 & $91 \cdot 73$ & $93 \cdot 20$ & 94.69 & 91.48 & $<0.0001$ \\
\hline Recognition of the European label (yes) (\%)† & $26 \cdot 84$ & $22 \cdot 73$ & $46 \cdot 82$ & $68 \cdot 24$ & $28 \cdot 35$ & $<0.0001$ \\
\hline Self-reported organic food consumption (\%)† & & & & & & $<0.0001$ \\
\hline Non-consumers & $17 \cdot 54$ & 28.50 & 0.55 & $0 \cdot 12$ & $10 \cdot 17$ & \\
\hline Occasional consumers & 76.96 & 69.33 & $48 \cdot 11$ & $8 \cdot 00$ & 73.65 & \\
\hline Regular consumers & $5 \cdot 50$ & $2 \cdot 17$ & $51 \cdot 34$ & 91.87 & $16 \cdot 17$ & \\
\hline
\end{tabular}

${ }^{*} P$ values are based on the $t$ test or $\chi^{2}$ test as appropriate.

† Because of missing data, the sample size was 23010 (cluster $1=9003$, cluster $2=4839$, cluster $3=5606$, cluster $4=2412$, cluster $5=1150$ ).

(high PANDiet and mPNNS-GS) (Table 2). Subjects had intermediate-to-high prevalence of inadequate intakes (Table 3). They reported high diversity in their places of purchase with the supermarket reported as the most frequent place of purchase followed by organic food stores and markets (Table 4). The cluster has the second highest percentage of subjects reporting environmentally friendly practices (Table 5).

Cluster 4: 'green' organic food eaters. This group comprised high organic food consumers, who defined themselves mostly as regular organic food consumers, and had $14 \%$ of vegetarians and vegans (Table 1). They were often highly educated and were largely able to recognise the official labels. The highest proportion of individuals living in rural areas was found in this cluster. Subjects also had the highest contribution of carbohydrate and the lowest contribution of protein to the total energy intake and the highest intakes of PUFA (Table 2). The highest PANDiet and mPNNS-GS scores were found in this cluster. They also exhibited the lowest prevalence of inadequate intakes of folate, vitamin $\mathrm{C}$, vitamin $\mathrm{E}, \mathrm{Mg}$ and $\mathrm{Fe}$ and the highest prevalence of inadequate intake of vitamin $\mathrm{B}_{12}$ (Table 3 ). They also favoured specialised organic food stores and short food supply chains (such as associations supporting small producers) as places of purchase (Table 4). The highest percentage of subjects with environmentally friendly practices was found in this cluster (Table 5).

Cluster 5: hedonist moderate organic food eaters. This cluster comprised a high proportion of men, individuals with high income and smokers (Table 1). Subjects were also older compared with other clusters. This cluster was mainly characterised by the particularly high intake of proteins and ethanol and a moderate consumption of organic food (Table 2). Overall, they exhibited poor nutritional diet quality with low PANDiet and mPNNS-GS scores. Subjects had the highest prevalence of inadequate intakes of $\mathrm{Ca}$ (Table 3). Although this group did the majority of their food purchasing in supermarkets, they also visited retail stores more frequently compared with other groups (Table 4).

\section{Discussion}

To our knowledge, no previous study has integrated both food groups and modes of food production in a cluster analysis. In our population, we identify five major groups of consumers: two major groups of low or very low organic food consumers (clusters 1 and 2), two groups of consumers with a high proportion of organic food in their diet (clusters 3 and 4) and a 
Table 2. Daily intakes of the thirty-two food groups included in the principal component analysis (PCA) and nutritional profiles across clusters, NutriNet-Sante study, 2014 (Mean values and $95 \%$ confidence intervals; $n$ 28245)

\begin{tabular}{|c|c|c|c|c|c|c|c|c|c|c|c|}
\hline \multirow[b]{3}{*}{ Labels } & \multicolumn{2}{|c|}{ Cluster 1 ( $n$ 11012) } & \multicolumn{2}{|c|}{ Cluster 2 ( $n$ 5811) } & \multicolumn{2}{|c|}{ Cluster 3 ( $n$ 7007) } & \multicolumn{2}{|c|}{ Cluster 4 (n 3048) } & \multicolumn{2}{|c|}{ Cluster 5 ( $n$ 1367) } & \multirow[b]{3}{*}{$P^{*}$} \\
\hline & \multicolumn{2}{|c|}{$\begin{array}{l}\text { Standard conventional food } \\
\text { small eaters }\end{array}$} & \multicolumn{2}{|c|}{$\begin{array}{l}\text { Unhealthy conventional food } \\
\text { big eaters }\end{array}$} & \multicolumn{2}{|c|}{$\begin{array}{l}\text { Standard organic food } \\
\text { small eaters }\end{array}$} & \multicolumn{2}{|c|}{ Green organic food eaters } & \multicolumn{2}{|c|}{$\begin{array}{l}\text { Hedonist moderate organic } \\
\text { food eaters }\end{array}$} & \\
\hline & Mean & $95 \% \mathrm{Cl}$ & Mean & $95 \% \mathrm{Cl}$ & Mean & $95 \% \mathrm{Cl}$ & Mean & $95 \% \mathrm{Cl}$ & Mean & $95 \% \mathrm{Cl}$ & \\
\hline \multicolumn{12}{|l|}{ Construction variables $\dagger$} \\
\hline \multicolumn{12}{|l|}{ Organic food intake $(\mathrm{g} / \mathrm{d}$ or $\mathrm{ml} / \mathrm{d})$} \\
\hline $\begin{array}{l}\text { Fruits and vegetables } \\
\text { (including juices and soups) }\end{array}$ & $114 \cdot 77$ & $110 \cdot 04,119.50$ & $89 \cdot 49 \ddagger$ & $82.98,96 \cdot 00 \ddagger$ & $435 \cdot 74$ & $429 \cdot 81,441 \cdot 67$ & $819.99 \ddagger$ & $811 \cdot 0,828 \cdot 97 \ddagger$ & $214 \cdot 28$ & $200 \cdot 85,227 \cdot 70$ & $<0.0001$ \\
\hline Seafood & 3.27 & $2.96,3.57$ & $2 \cdot 76 \ddagger$ & $2 \cdot 34,3 \cdot 18 \ddagger$ & $15 \cdot 36$ & $14.97,15.74$ & $17 \cdot 45 \ddagger$ & $16.87,18.03 \ddagger$ & 11.32 & $10 \cdot 45,12 \cdot 18$ & $<0.0001$ \\
\hline Meat, poultry, processed meat & $10 \cdot 93 \ddagger$ & $10.35,11.52 \ddagger$ & 11.07 & $10.26,11.87$ & $41 \cdot 16 \ddagger$ & $40.43,41.89 \ddagger$ & 40.23 & $39.12,41.34$ & 31.81 & $30.15,33.46$ & $<0.0001$ \\
\hline Eggs & $3.65 \ddagger$ & $3.48,3.82 \ddagger$ & 4.16 & $3.93,4.40$ & $10 \cdot 05$ & $9 \cdot 84,10 \cdot 26$ & $13.74 \ddagger$ & $13.41,14.06 \ddagger$ & 6.68 & $6 \cdot 20,7 \cdot 16$ & $<0.0001$ \\
\hline Dairy products & $32 \cdot 82$ & $30.90,34.73$ & $24 \cdot 17 \ddagger$ & $21 \cdot 53,26 \cdot 81 \ddagger$ & $127 \cdot 37 \ddagger$ & $124 \cdot 97,129 \cdot 77 \ddagger$ & 122.84 & $119 \cdot 19,126 \cdot 48$ & 50.49 & $45.05,55.94$ & $<0.0001$ \\
\hline Starchy foods & 19.77 & $18.79,20.75$ & $16 \cdot 29 \ddagger$ & $14.94,17.63 \ddagger$ & $71 \cdot 16$ & $69.93,72.38$ & $154.39 \ddagger$ & $152 \cdot 53,156 \cdot 26 \ddagger$ & $41 \cdot 17$ & $38.39,43.95$ & $<0.0001$ \\
\hline Whole-grain products & 9.52 & $8.69,10.35$ & $6 \cdot 24 \ddagger$ & $5 \cdot 10,7 \cdot 38 \ddagger$ & $42 \cdot 48$ & $41.44,43.52$ & $105 \cdot 47 \ddagger$ & $103 \cdot 89,107 \cdot 05 \ddagger$ & 18.33 & $15.97,20.68$ & $<0.0001$ \\
\hline Oil & 3.37 & $3.18,3.56$ & $2.53 \ddagger$ & $2 \cdot 27,2 \cdot 79 \ddagger$ & 13.91 & $13.67,14.14$ & $28 \cdot 36 \ddagger$ & $28.00,28.72 \ddagger$ & 7.56 & $7.02,8.09$ & $<0.0001$ \\
\hline Butter/margarine & 0.66 & $0.60,0.72$ & $0.57 \ddagger$ & $0.49,0.66 \ddagger$ & 3.22 & $3.15,3.30$ & $4.62 \ddagger$ & $4.51,4.74 \ddagger$ & 1.88 & $1.71,2.06$ & $<0.0001$ \\
\hline Sweetened foods & 6.89 & $6 \cdot 55,7.24$ & $6 \cdot 25 \ddagger$ & $5 \cdot 77,6.73 \ddagger$ & $26 \cdot 03$ & $25 \cdot 60,26 \cdot 47$ & $46 \cdot 75 \ddagger$ & $46.09,47.42 \ddagger$ & 14.78 & $13.80,15.77$ & $<0.0001$ \\
\hline Alcoholic beverages & 4.98 & $4 \cdot 18,5 \cdot 78$ & $4.02 \ddagger$ & $2 \cdot 91,5 \cdot 12 \ddagger$ & 21.92 & $20.91,22.93$ & $47.81^{\circ}$ & $46.29,49 \cdot 34$ & $96.57 \ddagger$ & $94.29,98.85 \ddagger$ & $<0.0001$ \\
\hline Non-alcoholic drinks & $96 \cdot 27$ & $91 \cdot 11,101.42$ & $62.90 \ddagger$ & $55.80,70.00 \ddagger$ & 384.68 & $378 \cdot 21,391 \cdot 14$ & $714 \cdot 27 \ddagger$ & $704.47,724.07 \ddagger$ & 170.53 & $155 \cdot 89,185 \cdot 16$ & $<0.0001$ \\
\hline Fast food & 1.76 & $1.55,1.96$ & $1.52 \ddagger$ & $1.24,1.80 \ddagger$ & 9.63 & $9.37,9.88$ & $20 \cdot 38 \ddagger$ & $19.99,20.76 \ddagger$ & 5.59 & $5 \cdot 02,6 \cdot 17$ & $<0.0001$ \\
\hline $\begin{array}{l}\text { Extra food (including snacks, chips, } \\
\text { salted biscuits, dressing } \\
\text { and sauces) }\end{array}$ & $1 \cdot 20$ & $1.05,1.36$ & $1 \cdot 20 \ddagger$ & $0.98,1.41 \ddagger$ & $6 \cdot 36$ & $6 \cdot 16,6 \cdot 56$ & $20 \cdot 18 \ddagger$ & $19 \cdot 88,20 \cdot 48 \ddagger$ & 3.59 & $3.14,4.04$ & $<0.0001$ \\
\hline $\begin{array}{l}\text { Dairy products and meat substitutes } \\
\text { (including soya-based products) }\end{array}$ & $9 \cdot 21$ & $7 \cdot 74,10 \cdot 67$ & $5 \cdot 44 \ddagger$ & $3.43,7.46 \ddagger$ & 24.56 & $22 \cdot 72,26 \cdot 39$ & $130 \cdot 22 \ddagger$ & $127.44,133.00 \ddagger$ & $6 \cdot 31$ & $2 \cdot 16,10 \cdot 47$ & $<0.0001$ \\
\hline $\begin{array}{l}\text { Other fats (including mayonnaise, } \\
\text { fresh cream, vegetable fresh } \\
\text { cream) }\end{array}$ & $0.28 \ddagger$ & $0.23,0.32 \ddagger$ & 0.33 & $0.27,0.39$ & 1.44 & $1 \cdot 38,1.49$ & $4 \cdot 14 \ddagger$ & $4.06,4.23 \ddagger$ & 0.63 & $0.50,0.75$ & $<0.0001$ \\
\hline \multicolumn{12}{|l|}{ Conventional food intake $(\mathrm{g} / \mathrm{d}$ or $\mathrm{ml} / \mathrm{d}$ ) } \\
\hline $\begin{array}{l}\text { Fruits and vegetables } \\
\text { (including juices and soups) }\end{array}$ & $477 \cdot 17$ & $471 \cdot 34,483 \cdot 00$ & $730 \cdot 31 \ddagger$ & $722 \cdot 29,738 \cdot 34 \ddagger$ & 333.67 & $326 \cdot 36,340 \cdot 98$ & $195 \cdot 01 \ddagger$ & $183 \cdot 93,206 \cdot 09 \ddagger$ & 429.67 & $413 \cdot 12,446 \cdot 22$ & $<0.0001$ \\
\hline Seafood & 32.76 & $32.02,33.49$ & $55.57 \ddagger$ & $54.56,56.59 \ddagger$ & 33.91 & $32.99,34.83$ & $30 \cdot 19 \ddagger$ & $28.79,31.59 \ddagger$ & 44.83 & $42 \cdot 75,46 \cdot 92$ & $<0.0001$ \\
\hline Meat, poultry, processed meat & 85.62 & $84.43,86.82$ & $163.02 \ddagger$ & $161 \cdot 37,164 \cdot 67 \ddagger$ & 56.95 & $55.45,58.45$ & $28.67 \ddagger$ & $26 \cdot 40,30.95 \ddagger$ & $122 \cdot 17$ & $118 \cdot 77,125 \cdot 57$ & $<0.0001$ \\
\hline Eggs & 4.74 & $4.59,4.89$ & $10 \cdot 46 \ddagger$ & $10 \cdot 25,10 \cdot 67 \ddagger$ & 1.89 & $1.70,2.08$ & $0.85 \ddagger$ & $0.56,1.13 \ddagger$ & 5.63 & $5.20,6.06$ & $<0.0001$ \\
\hline Dairy products & 205.75 & $202 \cdot 74,208 \cdot 77$ & $308.23 \ddagger$ & $304.08,312 \cdot 38 \ddagger$ & 112.54 & $108 \cdot 76,116 \cdot 32$ & $36.50 \ddagger$ & $30 \cdot 77,42 \cdot 23 \ddagger$ & $138 \cdot 77$ & $130 \cdot 22,147 \cdot 33$ & $<0.0001$ \\
\hline Starchy foods & $129 \cdot 37$ & $127.80,130.94$ & $214.41 \ddagger$ & $212 \cdot 24,216 \cdot 57 \ddagger$ & 78.84 & $76.87,80 \cdot 81$ & $34.90 \ddagger$ & $31.91,37.88 \ddagger$ & $142 \cdot 11$ & $137.65,146.56$ & $<0.0001$ \\
\hline Whole-grain products & $30 \cdot 11$ & $29.27,30.94$ & $37.92 \ddagger$ & $36 \cdot 77,39.07 \ddagger$ & 24.82 & $23 \cdot 77,25 \cdot 87$ & $11.03 \ddagger$ & $9.44,12.62 \ddagger$ & 34.98 & $32.61,37.36$ & $<0.0001$ \\
\hline Oil & 10.94 & $10 \cdot 73,11 \cdot 14$ & $20.50 \ddagger$ & $20 \cdot 22,20 \cdot 78 \ddagger$ & 6.48 & $6 \cdot 23,6 \cdot 74$ & $2.68 \ddagger$ & $2 \cdot 29,3.07 \ddagger$ & $12 \cdot 11$ & $11.53,12 \cdot 69$ & $<0.0001$ \\
\hline Butter/margarine & 4.71 & $4.60,4.81$ & $9 \cdot 10 \ddagger$ & $8.96,9.24 \ddagger$ & $2 \cdot 66$ & $2.53,2.79$ & $0.91 \ddagger$ & $0.71,1 \cdot 10 \ddagger$ & 5.89 & $5 \cdot 59,6 \cdot 18$ & $<0.0001$ \\
\hline Sweetened foods & $52 \cdot 17$ & $51.32,53.02$ & $93.28 \ddagger$ & $92 \cdot 11,94.45 \ddagger$ & 36.94 & $35.87,38.00$ & $22.68 \ddagger$ & $21 \cdot 06,24.29 \ddagger$ & 51.42 & $49.01,53.83$ & $<0.0001$ \\
\hline Alcoholic beverages & 61.41 & $59.36,63.45$ & $101 \cdot 28$ & $98.46,104.09$ & $55 \cdot 12$ & $52.56,57.68$ & $43.94 \ddagger$ & $40.05,47.83 \ddagger$ & $427 \cdot 67 \ddagger$ & $421 \cdot 86,433.48 \ddagger$ & $<0.0001$ \\
\hline Non-alcoholic drinks & 1525.02 & $1511.82,1538.22$ & $1883 \cdot 29 \ddagger$ & $1865 \cdot 11,1901 \cdot 46 \ddagger$ & $1383 \cdot 20$ & $1366.65,1399 \cdot 74$ & $1118.82 \ddagger$ & $1093 \cdot 73,1143.92 \ddagger$ & 1413.02 & $1375.55,1450.49$ & $<0.0001$ \\
\hline Fast food & $25 \cdot 79$ & $25 \cdot 21,26 \cdot 38$ & $44.64 \ddagger$ & $43.84,45.45 \ddagger$ & $19 \cdot 25$ & $18.52,19.98$ & $13.85 \ddagger$ & $12 \cdot 74,14.96 \ddagger$ & 31.55 & $29.89,33.20$ & $<0.0001$ \\
\hline $\begin{array}{l}\text { Extra food (including snacks, chips, } \\
\text { salted biscuits, dressing } \\
\text { and sauces) }\end{array}$ & $10 \cdot 46$ & $10 \cdot 23,10 \cdot 68$ & $22 \cdot 12 \ddagger$ & $21 \cdot 81,22 \cdot 43 \ddagger$ & 8.42 & $8 \cdot 14,8 \cdot 70$ & $6.02 \ddagger$ & $5.59,6.44 \ddagger$ & 16.04 & $15.41,16.67$ & $<0.0001$ \\
\hline $\begin{array}{l}\text { Dairy products and meat } \\
\text { substitutes (including soya- } \\
\text { based products) }\end{array}$ & $6 \cdot 28$ & $5 \cdot 68,6 \cdot 88$ & $6 \cdot 84$ & $6.01,7.67$ & $5 \cdot 10$ & $4 \cdot 35,5 \cdot 86$ & $9.54 \ddagger$ & $8 \cdot 39,10 \cdot 68 \ddagger$ & $3.01 \ddagger$ & $1 \cdot 30,4 \cdot 72 \ddagger$ & $<0.0001$ \\
\hline
\end{tabular}




\section{N British Journal of Nutrition}

\begin{tabular}{|c|c|c|c|c|c|c|c|c|c|c|c|}
\hline \multirow[b]{3}{*}{ Labels } & \multirow{2}{*}{\multicolumn{2}{|c|}{$\begin{array}{l}\text { Cluster } 1(n 11012) \\
\begin{array}{l}\text { andard conventional food } \\
\text { small eaters }\end{array}\end{array}$}} & \multirow{2}{*}{\multicolumn{2}{|c|}{ 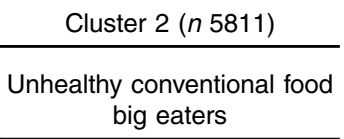 }} & \multirow{2}{*}{\multicolumn{2}{|c|}{$\begin{array}{c}\text { Cluster } 3(n \text { 7007) } \\
\begin{array}{c}\text { Standard organic food } \\
\text { small eaters }\end{array}\end{array}$}} & \multicolumn{2}{|c|}{ Cluster 4 ( $n$ 3048) } & \multicolumn{2}{|c|}{ Cluster 5 (n 1367) } & \multirow[b]{3}{*}{$P^{*}$} \\
\hline & & & & & & & \multicolumn{2}{|c|}{ Green organic food eaters } & \multicolumn{2}{|c|}{$\begin{array}{l}\text { Hedonist moderate organic } \\
\text { food eaters }\end{array}$} & \\
\hline & Mean & $95 \% \mathrm{Cl}$ & Mean & $95 \% \mathrm{Cl}$ & Mean & $95 \% \mathrm{Cl}$ & Mean & $95 \% \mathrm{Cl}$ & Mean & $95 \% \mathrm{Cl}$ & \\
\hline $\begin{array}{l}\text { Other fats (including mayonnaise, } \\
\text { fresh cream, vegetable fresh } \\
\text { cream) }\end{array}$ & 2.07 & $2 \cdot 00,2 \cdot 14$ & $5 \cdot 15 \ddagger$ & $5 \cdot 06,5 \cdot 25 \ddagger$ & 1.48 & $1.39,1.56$ & $0.76 \neq$ & $0.63,0.89 \ddagger$ & $2 \cdot 08$ & $1 \cdot 88,2 \cdot 27$ & $<0.0001$ \\
\hline \multicolumn{12}{|l|}{ Nutritional descriptive variables§ } \\
\hline $\begin{array}{l}\text { Proportion of organic food } \\
\text { in the diet }(\%) \|\end{array}$ & $13 \cdot 66$ & $13.31,14.03$ & $8.59 \ddagger$ & $8 \cdot 14,9.05 \ddagger$ & $50 \cdot 30$ & $49.89,50 \cdot 71$ & $78.53 \ddagger$ & $77 \cdot 98,79 \cdot 10 \ddagger$ & $25 \cdot 27$ & $24 \cdot 5,26 \cdot 05$ & $<0.0001$ \\
\hline Total energy intake $(\mathrm{kJ} / \mathrm{d})$ & $7104 \ddagger$ & $7050,7159 \ddagger$ & $10723 \ddagger$ & $10657,10786 \ddagger$ & 8163 & 8096,8226 & 9895 & 9811,9979 & 9598 & 9477,9715 & \\
\hline Total energy intake (kcal/d) & $1698 \ddagger$ & $1685,1711 \ddagger$ & $2563 \ddagger$ & $2547,2578 \ddagger$ & 1951 & 1935, 1966 & 2365 & 2345,2385 & 2294 & 2265,2322 & $<0.0001$ \\
\hline Lipids (\% of total energy intake) & $38.86 \ddagger$ & $38.68,39.04 \ddagger$ & 40.54 & $40 \cdot 31,40 \cdot 77$ & 40.43 & $40 \cdot 23,40 \cdot 64$ & 41.91‡ & $41 \cdot 63,42 \cdot 19 \ddagger$ & 41.77 & $41 \cdot 38,42 \cdot 17$ & $<0.0001$ \\
\hline $\begin{array}{l}\text { Carbohydrates } \\
\text { (\% of total energy intake) }\end{array}$ & $41 \cdot 78$ & $41.59,41.98$ & $39 \cdot 85$ & $39.61,40 \cdot 10$ & 40.89 & $40 \cdot 67,41 \cdot 11$ & $41 \cdot 87 \ddagger$ & $41 \cdot 57,42 \cdot 17 \ddagger$ & $38.46 \ddagger$ & $38 \cdot 04,38 \cdot 88 \ddagger$ & $<0.0001$ \\
\hline $\begin{array}{l}\text { Total simple carbohydrates } \\
\text { (\% of total energy intake) }\end{array}$ & $20 \cdot 71 \ddagger$ & $20 \cdot 55,20 \cdot 36 \ddagger$ & $19 \cdot 85$ & $19.64,20.06$ & 20.55 & $20 \cdot 36,20 \cdot 73$ & $20 \cdot 51$ & $20 \cdot 26,20 \cdot 77$ & $17 \cdot 84 \ddagger$ & $17 \cdot 48,18 \cdot 19 \ddagger$ & $<0.0001$ \\
\hline Protein (\% of total energy intake) & 18.95 & $18.86,19.04$ & $19 \cdot 21$ & $19 \cdot 10,19 \cdot 33$ & $18 \cdot 29$ & $18 \cdot 19,18.40$ & $15 \cdot 86 \ddagger$ & $15 \cdot 72,16 \cdot 01 \ddagger$ & $19 \cdot 42 \ddagger$ & $19 \cdot 22,19 \cdot 62 \ddagger$ & $<0.0001$ \\
\hline Fibre $(\mathrm{g} / \mathrm{d})$ & 21.35 & $21 \cdot 15,21 \cdot 55$ & 19.83 & $19.57,20.09$ & 23.89 & $23 \cdot 66,24 \cdot 13$ & $31.09 \ddagger$ & $30 \cdot 77,31 \cdot 41 \ddagger$ & $17 \cdot 21 \ddagger$ & $16 \cdot 77,17 \cdot 65 \ddagger$ & $<0.0001$ \\
\hline PUFA (g/d) & 12.93 & $12.78,13.07$ & 13.92 & $13 \cdot 74,14 \cdot 10$ & 14.38 & $14.22,14.54$ & $19.89 \ddagger$ & $19 \cdot 67,20 \cdot 11 \ddagger$ & $12 \cdot 43 \ddagger$ & $12 \cdot 13,12 \cdot 74 \ddagger$ & $<0.0001$ \\
\hline MUFA (g/d) & 32.54 & $32 \cdot 31,32 \cdot 77$ & 34.17 & $33.88,34.46$ & 34.63 & $34.37,34.90$ & $38.60 \ddagger$ & $38.24,38.95 \ddagger$ & $32.43 \ddagger$ & $31.93,32.92 \ddagger$ & $<0.0001$ \\
\hline SFA (g/d) & $33 \cdot 19$ & $32.98,33.40$ & $33 \cdot 40 \ddagger$ & $33 \cdot 13,33 \cdot 67 \ddagger$ & 32.00 & $31 \cdot 75,32 \cdot 24$ & $26 \cdot 15 \ddagger$ & $25 \cdot 82,26 \cdot 48 \ddagger$ & $29 \cdot 68$ & $29 \cdot 22,30 \cdot 14$ & $<0.0001$ \\
\hline$n-3$ Fatty acids $(g / d)$ & 1.70 & $1.67,1.73$ & 1.70 & $1.66,1.74$ & 2.08 & $2 \cdot 04,2 \cdot 12$ & $3.05 \ddagger$ & $3.00,3 \cdot 10 \ddagger$ & $1.61 \ddagger$ & $1.54,1.68 \ddagger$ & $<0.0001$ \\
\hline$n-6$ Fatty acids $(g / d)$ & 10.50 & $10.37,10.63$ & 11.45 & $11.29,11.61$ & 11.59 & $11.44,11.73$ & $16 \cdot 20 \ddagger$ & $16.00,16.40 \ddagger$ & $10 \cdot 12 \ddagger$ & $9 \cdot 85,10 \cdot 39 \ddagger$ & $<0.0001$ \\
\hline Cholesterol (mg/d) & 292.83 & $290 \cdot 24,295 \cdot 41$ & $327 \cdot 38 \ddagger$ & $324 \cdot 11,330 \cdot 66 \ddagger$ & $287 \cdot 33$ & $284 \cdot 38,290 \cdot 28$ & $206 \cdot 22 \ddagger$ & $202 \cdot 23,210 \cdot 22 \ddagger$ & 287.69 & $282 \cdot 12,293 \cdot 25$ & $<0.0001$ \\
\hline Ethanol $(\mathrm{g} / \mathrm{d})$ & 7.41 & $7 \cdot 17,7 \cdot 66$ & 7.80 & $7.49,8 \cdot 11$ & 7.44 & $7 \cdot 17,7 \cdot 72$ & $7.40 \ddagger$ & $7 \cdot 02,7 \cdot 77 \ddagger$ & $40 \cdot 60 \ddagger$ & $40.07,41.13 \ddagger$ & $<0.0001$ \\
\hline PANDiet & 70.06 & $69.87,70.26$ & $68 \cdot 70 \ddagger$ & $68.45,68.95 \ddagger$ & 71.06 & $70.84,71.28$ & $73.37 \ddagger$ & $73 \cdot 06,73.67 \ddagger$ & $69 \cdot 30$ & $68.88,69.72$ & $<0.0001$ \\
\hline mPNNS-GS & $8 \cdot 26$ & $8 \cdot 22,8 \cdot 30$ & $8 \cdot 22$ & $8 \cdot 17,8 \cdot 27$ & 8.71 & $8 \cdot 66,8 \cdot 76$ & $8 \cdot 87 \ddagger$ & $8.81,8.93 \ddagger$ & $7 \cdot 71 \ddagger$ & $7 \cdot 62,7 \cdot 80 \ddagger$ & $<0.0001$ \\
\hline
\end{tabular}

mPNNS-GS, modified Programme National Nutrition Santé Guideline Score.

* ANCOVA was used for testing differences between clusters.

† Thirty-two variables included in the PCA.

$\mp$ Values denote the highest and the lowest values.

$\S$ Adjusted for sex, age and total energy intake.

॥ Proportion of organic food in the diet: average percentages computed by averaging the total organic food intake $(\mathrm{g} / \mathrm{d})$ out of the total intake excluding water $(\mathrm{g} / \mathrm{d})$ multiplied by 100 
Table 3. Prevalence of inadequate intakes based on estimated average requirement (EAR) across clusters, NutriNet-Santé study, $2014(n$ 28245)*

\begin{tabular}{|c|c|c|c|c|c|c|}
\hline \multirow[b]{2}{*}{ Labels } & \multirow{2}{*}{$\begin{array}{c}\text { Cluster } 1 \text { ( } n \text { 11012) } \\
\begin{array}{c}\text { Standard } \\
\text { conventional food } \\
\text { small eaters }\end{array}\end{array}$} & \multirow{2}{*}{$\begin{array}{c}\text { Cluster } 2 \text { ( } n \text { 5811) } \\
\text { Unhealthy } \\
\begin{array}{c}\text { conventional food } \\
\text { big eaters }\end{array}\end{array}$} & \multirow{2}{*}{$\begin{array}{l}\text { Cluster } 3 \text { ( } n 7007) \\
\text { Standard organic } \\
\text { food small eaters }\end{array}$} & \multirow{2}{*}{$\begin{array}{l}\text { Cluster } 4 \text { (n 3048) } \\
\begin{array}{c}\text { Green organic } \\
\text { food eaters }\end{array}\end{array}$} & \multirow{2}{*}{$\begin{array}{l}\text { Cluster } 5 \text { ( } n \text { 1367) } \\
\text { Hedonist moderate } \\
\text { organic food eaters }\end{array}$} & \multirow[b]{2}{*}{$P+$} \\
\hline & & & & & & \\
\hline Retinol & $8 \cdot 33 \ddagger$ & $0.53 \ddagger$ & $3 \cdot 27$ & 1.48 & 5.41 & $<0.0001$ \\
\hline Thiamin & $22.07 \ddagger$ & $1.27 \ddagger$ & $12 \cdot 74$ & 2.56 & 14.41 & $<0.0001$ \\
\hline Riboflavin & $9.05 \ddagger$ & $0.29 \ddagger$ & 6.42 & 2.92 & 2.56 & $<0.0001$ \\
\hline Niacin & $2 \cdot 62 \ddagger$ & $0.07 \ddagger$ & 1.56 & 0.59 & 0.66 & $<0.0001$ \\
\hline Pantothenic acid & $13.85 \ddagger$ & $0.59 \ddagger$ & $10 \cdot 13$ & $3 \cdot 38$ & $3 \cdot 15$ & $<0.0001$ \\
\hline Vitamin $B_{6}$ & $28.90 \ddagger$ & $2.43 \ddagger$ & $14 \cdot 67$ & 3.28 & $10 \cdot 53$ & $<0.0001$ \\
\hline Folate & $14.96 \ddagger$ & 1.31 & $5 \cdot 24$ & $0.49 \ddagger$ & 9.44 & $<0.0001$ \\
\hline Vitamin $B_{12}$ & 5.46 & $0.55 \ddagger$ & 5.55 & $12 \cdot 03 \ddagger$ & 1.39 & $<0.0001$ \\
\hline Vitamin C & $31.91 \ddagger$ & 12.89 & $19 \cdot 37$ & $8.52 \ddagger$ & $25 \cdot 60$ & $<0.0001$ \\
\hline Vitamin E & $40 \cdot 21 \ddagger$ & 3.70 & $19 \cdot 27$ & $2 \cdot 20 \ddagger$ & 24.07 & $<0.0001$ \\
\hline I & $17 \cdot 85 \ddagger$ & $1.50 \ddagger$ & 9.70 & 5.87 & 9.29 & $<0.0001$ \\
\hline $\mathrm{Mg}$ & $34.65 \ddagger$ & 12.01 & $17 \cdot 20$ & $3.02 \ddagger$ & $17 \cdot 78$ & $<0.0001$ \\
\hline $\mathrm{P}$ & $1.55 \ddagger$ & $0.02 \ddagger$ & 1.20 & 0.13 & 0.51 & $<0.0001$ \\
\hline $\mathrm{Fe}$ & $37 \cdot 71 \ddagger$ & 7.06 & $19 \cdot 47$ & $3.02 \ddagger$ & 3.37 & $<0.0001$ \\
\hline $\mathrm{Ca}$ & $3.51 \ddagger$ & $8 \cdot 19$ & 29.56 & 20.95 & $31.46 \ddagger$ & $<0.0001$ \\
\hline $\mathrm{Zn}$ & $4.80 \ddagger$ & $0.10 \ddagger$ & 3.81 & 0.89 & 1.68 & $<0.0001$ \\
\hline $\mathrm{K}$ & $20.39 \ddagger$ & $1 \cdot 12 \ddagger$ & 10.95 & 2.75 & $4 \cdot 17$ & $<0.0001$ \\
\hline $\mathrm{Se}$ & $11.40 \ddagger$ & $0.46 \ddagger$ & 6.58 & $2 \cdot 13$ & $7 \cdot 10$ & $<0.0001$ \\
\hline
\end{tabular}

* Prevalence of inadequate intakes $=$ proportion of the subjects with intake below the EAR.

$\dagger P$ values are based on $\chi^{2}$ test.

$\ddagger$ Values denote the highest and the lowest values.

group with a moderate consumption of organic food (cluster 5). Their profiles in terms of socio-demographic, behavioural and nutrient characteristics were markedly different, demonstrating a variety of eating habits and patterns. In general, high levels of organic food consumption were associated with an overall better nutritional diet quality. Overall, the total energy intake, the nutritional diet quality, but also the modes of food production emerged as major discriminating components.

\section{Socio-demographic and lifestyle characteristics}

Socio-demographic and lifestyle differences between nonorganic food consumers and frequent organic food consumers are in line with those observed in other populations $(2,3,5,6,13,15)$ and in the NutriNet-Santé cohort in particular ${ }^{(12)}$. Our findings also corroborate the fact that, in general, a higher education level seems to be a strong determinant of organic food consumption $^{(4,6,7,10,13)}$. Unlike previous research showing that organic food consumption tends to be positively related to urbanisation $^{(6,13)}$, the percentage of individuals living in a rural community is highest among the groups with the highest level of organic food consumption. Several hypothesises may explain why high organic food consumers reside more often in rural areas. Country-dwellers (particularly those included in the NutriNet-Santé study) may have a special linkage with food, land and soil, and organic foods have been associated with freshness, tradition and naturalness ${ }^{(30-32)}$. They may also have high access to short food supply chains, and therefore better access to 'fresh' organic foods.

Regarding lifestyle characteristics, our results are consistent with a recent study showing that frequent organic food consumers exhibit healthier lifestyles with fewer smokers than non-consumers (this study reported $80 \%$ of non-smokers among buyers of organic food $v .71 \%$ among non-buyers) ${ }^{(15)}$. A strong positive association between frequent organic food consumption and vegetarianism has also been reported in the literature $^{(2,13)}$, supported by our findings. In the present study, the proportion of vegetarians and vegans was $14 \%$ among the high organic food consumers (cluster 4), reflecting the fact that very high organic food consumption is probably often related to specific dietary practices.

\section{Dietary profiles and eating-related practices}

Across clusters, different eating behaviours are reflected by various levels of nutrient intakes, and thus occurrence of inadequate intakes. The corollary of the low energy intake in cluster 1 is high prevalence of inadequate intakes of most nutrients. The opposite is true for cluster 2 where high energy intake is followed by low prevalence of inadequate intakes of most nutrients. Despite this fact, cluster 2 exhibited the lowest PANDiet score due to their high intakes of cholesterol or SFA.

Unsurprisingly, the highest prevalence of inadequate intakes of vitamin $\mathrm{B}_{12}$ was observed in cluster 4 , reflecting the plantbased diet of the subjects. Thus, $12 \%$ of subjects had an inadequate intake in this group, which compares with a prevalence of nearly $0 \%$ in the French population ${ }^{(33)}$. It is also noteworthy that subjects in cluster 4 had the lowest prevalence of inadequate intake of Fe. This can be attributed to their very high consumption of fruits and vegetables, whole-grain products and soya-based products. These results are in line with those of a previous study showing a higher intake of $\mathrm{Fe}$ in vegetarians compared with their omnivorous counterparts ${ }^{(34)}$. For folate, vitamin $\mathrm{C}$ or $\mathrm{Mg}$, subjects in cluster 4 had the lowest 
prevalence of inadequate intakes. These three micronutrients are indeed found mainly in 'healthy' foods such as fruits and vegetables largely consumed by subjects in cluster 4. This seems consistent with other studies reporting that frequent organic food consumers had higher intakes of most minerals and vitamins than non-consumers ${ }^{(9,12,13,15)}$.

Cluster 3 can be considered as an organic cluster 1. In other words, cluster 3 is an 'organic mirror' of cluster 1. Indeed, cluster 3 showed great similarities to cluster 1 , especially for the proportion of women and for subjects with high school diploma. However, subjects in cluster 3 had higher intakes of most nutrients and an overall higher nutritional diet quality than those in cluster 1. In a German study, separate cluster analyses have been carried out for male purchasers and non-purchasers of organic food in order to identify typology of purchasers ${ }^{(14)}$. Consistent with our findings, the results revealed that, although the same types of consumers were found for both groups, male organic purchasers nevertheless exhibited specific dietary patterns with higher intakes of fruits and vegetables and lower intakes of meat and were more likely to be motivated by sustainability criteria. As also observed in other studies ${ }^{(9,13,15)}$, a positive relationship was found between higher organic food consumption and healthier food habits. In the present study, the highest PANDiet and mPNNS-GS scores are thus found in cluster 3 and cluster 4 - that is, among subjects with the highest level of organic food in their diet. Furthermore, they are more prone to purchasing their foods from a variety of supply chains. The proportion of subjects with environmentally friendly practices was also higher in cluster 3 .

Cluster 4 exhibited environmentally friendly behaviours through their choice of products (plant food) and through their choice of the mode of food production (organic instead of conventional), thus fitting with the concept of sustainable diet described by the $\mathrm{FAO}^{(35)}$. Moreover, in our study as in another ${ }^{(36)}$, they also tended to make purchases more frequently from short food supply chains and from small-scale producers than other consumers, thus favouring local production.

These environmentally friendly food behaviours seem to be translated into environmentally friendly practices. For instance, a high percentage of subjects recycle their organic waste, in line with a previous study reporting that increasing organic food consumption was related to environmentally friendly consumption behaviours such as recycling ${ }^{(30)}$. These 'sustainable habits' are reflected in the ethical reasoning behind organic food consumption ${ }^{(3,11,36)}$.

Cluster 5 gathered a minority of subjects (5\%). They can be seen as a group of 'bon vivants'. They have a traditional diet rich in meat and alcohol with moderate organic food consumption. They have an 'unhealthier' lifestyle overall, as they are more often smokers and have higher alcohol intake.

Our study presents major strengths that include an innovative approach in the implementation of the typology of organic and conventional food consumers, as well as a large participant population allowing for identification of a variety of food habits and practices. Several limitations should also be mentioned. Intake of fruits and vegetables in our study was particularly high compared with the figures of the French National ENNS (Etude nationale nutrition santé) survey ${ }^{(37)}$. As the Org-FFQ was 
Table 5. Environmentally friendly practices across clusters, NutriNet-Santé study, 2014 (n 23010)*

\begin{tabular}{|c|c|c|c|c|c|c|}
\hline \multirow[b]{2}{*}{ Labels } & $\begin{array}{l}\text { Cluster } 1 \\
(n \text { 9003) }\end{array}$ & $\begin{array}{l}\text { Cluster } 2 \\
(n \text { 4839) }\end{array}$ & $\begin{array}{l}\text { Cluster } 3 \\
(n 5606)\end{array}$ & $\begin{array}{l}\text { Cluster } 4 \\
(n 2412)\end{array}$ & $\begin{array}{l}\text { Cluster } 5 \\
(n 1150)\end{array}$ & \\
\hline & $\begin{array}{c}\text { Standard } \\
\text { conventional food } \\
\text { small eaters }\end{array}$ & $\begin{array}{c}\text { Unhealthy } \\
\text { conventional food } \\
\text { big eaters }\end{array}$ & $\begin{array}{l}\text { Standard organic } \\
\text { food small eaters }\end{array}$ & $\begin{array}{l}\text { Green } \\
\text { organic food } \\
\text { eaters }\end{array}$ & $\begin{array}{c}\text { Hedonist } \\
\text { moderate organic } \\
\text { food eaters }\end{array}$ & $P \dagger$ \\
\hline $\begin{array}{l}\text { Did you consider the energy performance } \\
\text { label when purchasing your refrigerator? }\end{array}$ & & & & & & $<0.0001$ \\
\hline Yes & $66 \cdot 17$ & $66 \cdot 71$ & 74.69 & $76 \cdot 53 \ddagger$ & $63.04 \ddagger$ & \\
\hline No & 33.83 & 33.29 & $25 \cdot 31$ & $23 \cdot 47 \ddagger$ & $36.96 \ddagger$ & \\
\hline Do you regularly defrost your refrigerator? (\%) & & & & & & $<0.0001$ \\
\hline Yes, manually & $32 \cdot 24$ & $31 \cdot 78$ & 31.97 & $33.33 \ddagger$ & $30 \cdot 43 \ddagger$ & \\
\hline Yes, I have an automatic defrost & $54 \cdot 19 \ddagger$ & 56.58 & $57 \cdot 58$ & $55 \cdot 10$ & $59 \cdot 39 \ddagger$ & \\
\hline No & $13.56 \ddagger$ & 11.63 & 10.45 & 11.57 & $10 \cdot 17 \ddagger$ & \\
\hline Do you use a lid when heating up water? (\%) & & & & & & $<0.0001$ \\
\hline Yes & 79.52 & $78 \cdot 30 \ddagger$ & $87 \cdot 60$ & $90 \cdot 38 \ddagger$ & 82.52 & \\
\hline No & $20 \cdot 48$ & $21.70 \ddagger$ & $12 \cdot 40$ & $9.62 \ddagger$ & 17.48 & \\
\hline Do you recycle organic waste? (\%) & & & & & & $<0.0001$ \\
\hline Yes & $46 \cdot 78 \ddagger$ & 47.63 & 63.56 & $70 \cdot 36 \ddagger$ & 58.43 & \\
\hline No & $53.22 \ddagger$ & $52 \cdot 37$ & 36.44 & $29 \cdot 64 \ddagger$ & 41.57 & \\
\hline
\end{tabular}

* Because of missing data, sample size was 23010.

$\dagger P$ values are based on $\chi^{2}$ test.

$\ddagger$ Values denote the highest and the lowest values.

administered between summer and early autumn (from June to October), a possible seasonal effect cannot be ruled out. This is also true for the share of organic food in the whole diet compared with the organic French market ${ }^{(38)}$. This may be due to the assessment tool itself (Org-FFQ), but it may also be a consequence of the profiles of the NutriNet-Santé participants who are volunteers in a nutrition cohort ${ }^{(39)}$. They exhibit not only specific socio-demographic characteristics ${ }^{(39)}$ but also probably a higher interest in nutritional and health-related issues. Thus, extrapolation from the results must be done with caution. In addition, respondents to the Org-FFQ were more likely to be interested in sustainability issues. It is noteworthy that the use of a unique composition database to assess nutrient intakes did not allow the consideration of some differences in nutrient contents between organic and conventional foods, as reported in three recent meta-analyses (e.g. content in antioxidant or $n-3$ fatty acids) ${ }^{(20-22)}$.

To conclude, these results indicate that organic or conventional food consumers cannot be considered as two single monolithic groups. Considering that conventional food consumption is systematically associated with a poor quality of diet compared with organic food consumption would be a simplistic approach in aetiological studies on the effects of organic food consumption. This has implications for future studies investigating the potential health impacts of organic food that should take into account all dimensions of dietary patterns. These findings also stress the need to foster the implementation of consistent targeted-group sustainable policies. Notably, in 2015, in France, sales have increased in every supply chain. At the same time, suppliers are seeking to reach out to consumers through diverse channels. As organic supply continues to diversify, so will the consumers. It is therefore crucial to delve further into the understanding of the motivations, perceptions, attitudes and purchase practices of the different types of consumers.

\section{Acknowledgements}

The authors thank all the people who helped carry out the NutriNet-Santé study and all the dedicated and conscientious volunteers. The authors especially thank Younes Esseddik, Mohand Ait-Oufella, Paul Flanzy, Yasmina Chelghoum, Véronique Gourlet, Nathalie Arnault, Fabien Szabo, Laurent Bourhis, Charlotte Voetglin and Cédric Agaesse.

The BioNutriNet project was supported by the French National Research Agency (Agence Nationale de la Recherche) in the context of the 2013 Programme de Recherche Systèmes Alimentaires Durables (ANR-13-ALID-0001). The NutriNet-Santé cohort study is funded by the following public institutions: Ministère de la Santé, Institut de Veille Sanitaire, Institut National de la Prévention et de l'Education pour la Santé, Institut National de la Santé et de la Recherche Médicale, Institut National de la Recherche Agronomique, Conservatoire National des Arts et Métiers and Paris 13 University. The funders had no role in study design, data collection and analysis, decision to publish or in the preparation of the manuscript.

The author contributions were as follows: S. H., P. G., E. K.-G., S. P., C. M., M. T. and D. L. conceived and designed the experiments. J. B., S. H., P. G., E. K.-G., S. P., C. M. and M. T. performed the experiments. J. B. and E. K.-G. analysed the data. J. B. wrote the paper. J. B., M. T., B. A., S. P., C. M., P. G., S. H., D. L. and E. K.-G. were involved in interpreting results and editing the manuscript. J. B. had primary responsibility for the final content. All the authors read and approved the final version of the manuscript.

There are no conflicts of interest.

\section{References}

1. The World of Organic Agriculture (2015) Statistics and Emerging Trends 2015. FiBL-IFOAM Report. Bonn: Research Institute of Organic Agriculture (FiBL), Frick and IFOAM Organics International. 
2. Schifferstein HNJ \& Oude Ophuis PAM (1998) Health-related determinants of organic food consumption in The Netherlands. Food Qual Prefer 9, 119-133.

3. Hughner RS, McDonagh P, Prothero A, et al. (2007) Who are organic food consumers? A compilation and review of why people purchase organic food. J Consum Behav 6, 94-110.

4. Hassan D, Monier Dilhan S, Nichele V, et al. (2009) Organic food consumption patterns in France. J Agric Food Ind Org 7, 1-23.

5. Dettmann RL \& Dimitri C (2009) Who's buying organic vegetables? Demographic characteristics of U.S. consumers. J Food Prod Mark 16, 79-91.

6. Torjusen H, Brantsæter AL, Haugen M, et al. (2010) Characteristics associated with organic food consumption during pregnancy; data from a large cohort of pregnant women in Norway. BMC Public Health 10, 775.

7. Pearson D, Henryks J \& Jones H (2011) Organic food: what we know (and do not know) about consumers. Renew Agric Food Syst 26, 171-177.

8. Dimitri C \& Dettmann RL (2012) Organic food consumers: what do we really know about them? Br Food J 114, 1157-1183.

9. Torjusen H, Lieblein G, Naes T, et al. (2012) Food patterns and dietary quality associated with organic food consumption during pregnancy; data from a large cohort of pregnant women in Norway. BMC Public Health 12, 612.

10. Oates L, Cohen M \& Braun L (2012) Characteristics and consumption patterns of Australian organic consumers. J Sci Food Agric 92, 2782-2787.

11. Bravo CP, Cordts A, Schulze B, et al. (2013) Assessing determinants of organic food consumption using data from the German National Nutrition Survey II. Food Qual Prefer 28, 60-70.

12. Kesse-Guyot E, Péneau S, Méjean C, et al. (2013) Profiles of organic food consumers in a large sample of French adults: results from the Nutrinet-Santé cohort study. PLOS ONE $\mathbf{8}$, e76998.

13. Petersen SB, Rasmussen MA, Strøm M, et al. (2013) Sociodemographic characteristics and food habits of organic consumers - a study from the Danish National Birth Cohort. Public Health Nutr 16, 1810-1819.

14. Cordts A, Wittig F, Schulze B, et al. (2013) A typology comparing male organic consumers and non-organic consumers: nutrition, health and buying behaviors. Ernährungs-Umschau 60, 36- 42 .

15. Eisinger-Watzl M, Wittig F, Heuer T, et al. (2015) Customers purchasing organic food - do they live healthier? Results of the German National Nutrition Survey II. Eur J Nutr Food Saf 5, 59-71.

16. Hercberg S, Castetbon K, Czernichow S, et al. (2010) The Nutrinet-Santé Study: a web-based prospective study on the relationship between nutrition and health and determinants of dietary patterns and nutritional status. BMC Public Health 10, 242.

17. Kesse-Guyot E, Castetbon K, Touvier M, et al. (2010) Relative validity and reproducibility of a food frequency questionnaire designed for French adults. Ann Nutr Metab 57, 153-162.

18. Baudry J, Méjean C, Allès B, et al. (2015) Contribution of organic food to the diet in a large sample of French adults (the NutriNet-Santé cohort study). Nutrients 7, 8615-8632.

19. Etude Nutrinet-Santé (2013) Table de Composition des Aliments de l'étude Nutrinet-Santé (Nutrinet-Santé Study Food Composition Database). Paris: Economica

20. Barański M, Średnicka-Tober D, Vola kakis N, et al. (2014) Higher antioxidant and lower cadmium concentrations and lower incidence of pesticide residues in organically grown crops: a systematic literature review and meta-analyses. Br J Nutr 112, 1-18.

21. Średnicka-Tober D, Barański M, Seal CJ, et al. (2016) Higher PUFA and $n$-3 PUFA, conjugated linoleic acid, $\alpha$-tocopherol and iron, but lower iodine and selenium concentrations in organic milk: a systematic literature review and meta- and redundancy analyses. Br J Nutr 115, 1043-1060.

22. Średnicka-Tober D, Barański M, Seal C, et al. (2016) Composition differences between organic and conventional meat: a systematic literature review and meta-analysis. Br J Nutr 115, 1-18.

23. Estaquio C, Kesse-Guyot E, Deschamps V, et al. (2009) Adherence to the French Programme National Nutrition Santé Guideline Score is associated with better nutrient intake and nutritional status. J Am Diet Assoc 109, 1031-1041.

24. Verger EO, Mariotti F, Holmes BA, et al. (2012) Evaluation of a diet quality index based on the probability of adequate nutrient intake (PANDiet) using national French and US dietary surveys. PLOS ONE 7, e42155.

25. Carriquiry AL (1999) Assessing the prevalence of nutrient inadequacy. Public Health Nutr 2, 23-34.

26. Martin A (2001) The 'apports nutritionnels conseilles (ANC)' for the French population. Reprod Nutr Dev 41, 119-128.

27. Institut National de la Statistique et des Etudes Economiques (2015) Definitions and methods. http://www.insee.fr/en/ methodes/ (accessed February 2015).

28. Schofield WN (1985) Predicting basal metabolic rate, new standards and review of previous work. Hum Nutr Clin Nutr 39, Suppl. 1, 5-41.

29. Hatcher L \& Stepanski EJ (1994) A Step-by-Step Approach to Using the SAS® System for Univariate and Multivariate Statistics. Cary, NC: SAS Institute.

30. Lockie S, Lyons K, Lawrence G, et al. (2004) Choosing organics: a path analysis of factors underlying the selection of organic food among Australian consumers. Appetite 43, 135-146.

31. Wier M, O'Doherty Jensen K, Andersen LM, et al. (2008) The character of demand in mature organic food markets: Great Britain and Denmark compared. Food Policy 33, 406-421.

32. Agence Bio/Conseil Sondage et Analyse (CSA) (2015) Baromètre de consommation et de perception des produits biologiques en France (Barometer of Consumption of Organic Products in France). Agence Bio CSA. http://www. agencebio.org/sites/default/files/upload/documents/4_Chiffres/ BarometreConso/barometre_agence_bio_public.pdf (accessed May 2015).

33. Touvier M, Lioret S, Vanrullen I, et al. (2006) Vitamin and mineral inadequacy in the French population: estimation and application for the optimization of food fortification. Int $J$ Vitam Nutr Res 76, 343-351.

34. Deriemaeker P, Alewaeters K, Hebbelinck M, et al. (2010) Nutritional status of flemish vegetarians compared with nonvegetarians: a matched samples study. Nutrients 2, 770-780.

35. Burlingame BA \& Dernini S (2012) Sustainable diets and biodiversity. In International Scientific Symposium Biodiversity and Sustainable Diets United against Hunger (2010: Rome, Italy). Rome: FAO.

36. Lockie S, Lyons K, Lawrence G, et al. (2002) Eating 'Green': motivations behind organic food consumption in Australia. Sociol Rural 42, 23-40.

37. Castetbon K, Vernay M, Malon A, et al. (2009) Dietary intake, physical activity and nutritional status in adults: the French nutrition and health survey (ENNS, 2006-2007). BrJ Nutr 102, 733-743.

38. Agence Bio (2014) La Bio en France: De la Production à la Consommation (Organic Food in France: From Production to Consumption). Agence Bio. http://www.agencebio.org/sites/ default/files/upload/documents/4_Chiffres/BrochureCC/ CC2013_chap4_France.pdf (accessed May 2015).

39. Andreeva VA, Salanave B, Castetbon K, et al. (2015) Comparison of the sociodemographic characteristics of the large NutriNetSanté e-cohort with French Census data: the issue of volunteer bias revisited. J Epidemiol Community Health 69, 893-898. 